\title{
Dynamics and Biocontrol: The Indirect Effects of a Predator Population on a Host-Vector Disease Model
}

\author{
Fengyan Zhou ${ }^{1,2}$ and Hongxing Yao ${ }^{1,3}$ \\ ${ }^{1}$ Faculty of Science, Jiangsu University, Zhenjiang, Jiangsu 212013, China \\ ${ }^{2}$ Department of Mathematics, Shaoxing University, Shaoxing, Zhejiang 312000, China \\ ${ }^{3}$ School of Finance and Economics, Jiangsu University, Zhenjiang, Jiangsu 212013, China
}

Correspondence should be addressed to Hongxing Yao; hxyao@ujs.edu.cn

Received 11 July 2013; Revised 29 November 2013; Accepted 10 December 2013; Published 27 January 2014

Academic Editor: Yanni Xiao

Copyright (C) 2014 F. Zhou and H. Yao. This is an open access article distributed under the Creative Commons Attribution License, which permits unrestricted use, distribution, and reproduction in any medium, provided the original work is properly cited.

\begin{abstract}
A model of the interactions among a host population, an insect-vector population, which transmits virus from hosts to hosts, and a vector predator population is proposed based on virus-host, host-vector, and prey (vector)-enemy theories. The model is investigated to explore the indirect effect of natural enemies on host-virus dynamics by reducing the vector densities, which shows the basic reproduction numbers $R_{01}$ (without predators) and $R_{02}$ (with predators) that provide threshold conditions on determining the uniform persistence and extinction of the disease in a host population. When the model is absent from predator, the disease is persistent if $R_{01}>1$; in such a case, by introducing predators of a vector, then the insect-transmitted disease will be controlled if $R_{02}<1$. From the point of biological control, these results show that an additional predator population of the vector may suppress the spread of vector-borne diseases. In addition, there exist limit cycles with persistence of the disease or without disease in presence of predators. Finally, numerical simulations are conducted to support analytical results.
\end{abstract}

\section{Introduction}

In recent years there has been an increasing attention of the importance of studying vector-host diseases, whereby diseases are not transmitted directly from hosts to hosts, but through a vector. For instance, human and animal diseases such as malaria, dengue fever, West Nile virus, and Lyme disease are transmitted by arthropod vectors. Also, plant virus diseases such as tobacco mosaic virus, African cassava mosaic virus, cucumber mosaic virus, potato virus $\mathrm{Y}$, beet yellows virus, and rice dwarf virus are transmitted by arthropod vectors. Bailey [1] pointed out that the vector population greatly influenced the modeling and control of infectious diseases. Moreover, vector-host diseases cause serious public health threat and great economic losses of agricultural production. Consequently, some effective control methods should be considered to prevent diseases transmitted by insect-vectors.

Generally, pesticide is the most used method of insecttransmitted diseases control. The abuse of pesticides exacerbates disease incidence by selecting insecticide resistant vectors [2] and heavily affects reproduction and predation behavior of the beneficial predators of the vector [3]. Moreover, the repeated use of pesticides will deteriorate environment and human health. Therefore, it is not reasonable to depend fully on insecticides for the control of vector populations.

The potential for predation to reduce disease prevalence in a host population also has implications for the biological control of vector populations. For example, in [4-11] predators have been introduced as biological control agents of vectors for various diseases such as malaria, dengue fever, and Lyme disease. Several recent studies suggested that predators led to a decline in local cases of dengue fever in Vietnam and Thailand $[12,13]$ and malaria in India $[14,15]$.

However, compared with biological control of herbivorous pests, which has long been established as a major component of pest management programmes and aims to direct decrease pest densities by pest enemies [16, 17], biological 
control of vectors has been seldom investigated. The main reason is that biological control of vectors is a complex interaction process, mainly including the virus-host interaction, vector-host interaction, and vector (prey)-enemy interaction, which is more complicated than the only pestenemy interaction [18-23] of biological pest control. The goal of biological control agents of vectors is to reduce disease incidence through the indirect action of natural enemy that lowers the density of a target vector population and is not necessarily to eliminate the vector [24]. The identification of a vector population threshold density plays an important role in determining whether predation could sufficiently reduce the vector population density [25]. Therefore, from the mathematical viewpoint, it is also important to investigate how vector (prey)-predator dynamics may be influenced by the additional vector mortality.

Previous results on vector-borne diseases mainly focused on the vector-host dynamics [26-34] or virus-host dynamics [35] without considering the vector-enemy dynamics. Only several authors have studied the biological control of vector to reduce the disease incidence. In $[36,37]$, different mathematical models are used to examine how Wolbachia affects the transmission of dengue based on the fact that Wolbachia reduces the lifespan of infected mosquito Aedes aegypti. Moore et al. [25] first proposed a host-vector-predator model and studied how the indirect effect of predators on vectors affects prevalence of vector-borne diseases. For plant virus diseases transmitted by arthropod vectors, Okamoto and Amarasekare [38] compared the efficacy of three types of biocontrol agents: predator/parasitoid, competitor, and pathogen of the vector (Whitefly, which transmits the African cassava mosaic virus from cassavas to cassavas) to reduce disease incidence. Both papers [25] and [38], however, focused on the indirect effect of natural enemies on the vector based on numerical analysis, explicit mathematical analysis has not been done in detail to obtain the threshold value determining the prevalence or extinction of disease, and more complex dynamics such as Hopf bifurcation in present of the vector predators have not been considered.

Motivated by the above mentioned surveys, the main objective of this study is to investigate the effects of predators on the prevalence of vector-borne disease by analyzing the stability of disease-free and disease equilibria in present of predators. Moreover, disease control threshold and limit cycles with persistence of disease or without disease in present of predators will be studied.

The rest of the paper is organized as follows. Section 2, we mainly formulate our basic mathematical model of hostvector-predator interactions. The equilibria and the reproduction number are given in Section 3. In Section 4, the local stability of the equilibria for model (2) is analyzed. In Section 5 , the global stability and Hopf bifurcation of model (2) are investigated; moreover, the control threshold is presented. In Section 6, we use some numerical simulations to explore the effect of predators on the persistence or eradication of virus transmitted by vectors, the stability results, and existence of limit cycles of our model with the predator population. Finally, concluding remarks are given in Section 7.

\section{Model Formulation}

In this section we mainly formulate a mathematical model to depict the interactions among a host population, a vector population, and a vector predator population.

First, we will give the following assumptions.

(1) The total host population $K(t)$ is divided into susceptible (healthy) and infected hosts, respectively, with densities denoted by $S(t)$ and $I(t)$, respectively, and the total vector population is also partitioned into susceptible and infectious subclasses, with the densities denoted by $X_{u}(t)$ and $X_{i}(t)$, respectively.

(2) Virus transmission is induced by vectors feeding on infected hosts or infected vectors feeding on susceptible hosts, without considering the direct transmission between susceptible and infected hosts.

(3) The virus in vectors may not cause vectors' death.

(4) The new born vectors are susceptible and unable to transmit virus from hosts to hosts.

(5) Vectors in both classes experience density-independent and density-dependent mortality in addition to death from predation.

(6) Vectors' predators are beneficial insects which can prey on vectors and may not damage hosts and environment, denoted by $P(t)$. The Holling type II functional [39] is used for interactions between vectors and predators.

(7) When there are no predators, we assume that the dynamics of the total vector population $X=X_{u}+X_{i}$ is governed by equation $d X / d t=r X(1-X / M-$ $m X /(S+I))$, where $r$ is the intrinsic growth rate, $M$ is vector's carrying capacity of the environment, and $m$ denotes the extent to which vectors are limited by the host population, and we assume that $m=0$ if the total host population on which the vectors depend keeps constant.

Our assumptions on the dynamical transfer of the host, the vector, and the predator population are demonstrated in Figure 1.

In Figure 1, $f(S, I)>0$ is the recruited rate of the host (or the replanting rate for the plant host) and $\mu$ and $d$ are the natural death rate and disease-induced mortality of the infected host $I$, respectively. $b_{1}(K)$ denotes the per capita rate of contacts on hosts by vectors. Generally, $b_{1}(K)$ is a monotonic increasing function on $K$. In this paper, we assume $b_{1}(K)=b_{1} K . \alpha$ and $\beta$ are the disease transmission coefficients from infected hosts to uninfected vectors and from infected vectors to uninfected hosts, respectively; $b, w$, and $r=b-w$ are the natural birth rate, the natural death rate and the intrinsic growth rate of vectors, respectively. The term $h X /(1+a X)$ denotes the Holling type II functional response of the predator. $h$ is the capturing rate (or the attacking rate) of predators, $a$ is predator's satiety rate, and $\gamma$ denotes the conversation factor of the predator, which describes the conversion efficiency of the consumed vectors into individual 


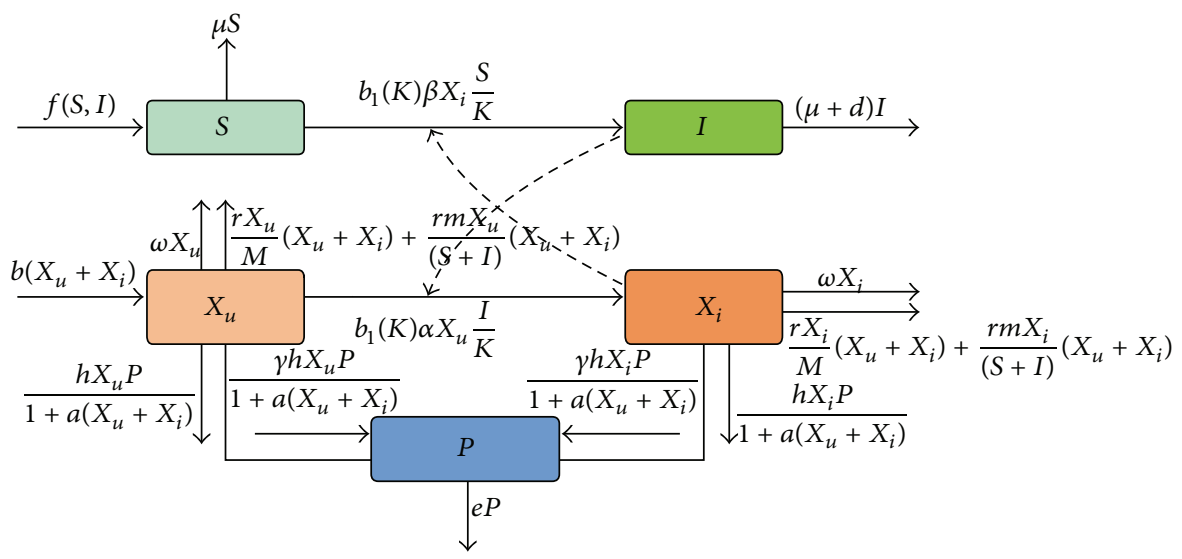

FIGURE 1: Flow chart of the host-vector-predator model.

predators. $e$ is the natural mortality of the predator. All the parameters above are positive.

Using the transfer diagram, the system which depicts the interactions among the host, the vector, and the predator population can be derived:

$$
\begin{gathered}
\frac{d S}{d t}=f(S, I)-b_{1}(K) \beta X_{i} \frac{S}{K}-\mu S, \\
\frac{d I}{d t}=b_{1}(K) \beta X_{i} \frac{S}{K}-(\mu+d) I, \\
\frac{d X_{u}}{d t}= \\
b\left(X_{u}+X_{i}\right)-b_{1}(K) \alpha X_{u} \frac{I}{K}-w X_{u} \\
-\frac{r X_{u}}{M}\left(X_{u}+X_{i}\right)-\frac{r m X_{u}}{(S+I)}\left(X_{u}+X_{i}\right) \\
-\frac{h X_{u}}{1+a\left(X_{u}+X_{i}\right)} P, \\
\frac{d X_{i}}{d t}=b_{1}(K) \alpha X_{u} \frac{I}{K}-w X_{i}-\frac{r X_{i}}{M}\left(X_{u}+X_{i}\right) \\
-\frac{r m X_{i}}{(S+I)}\left(X_{u}+X_{i}\right)-\frac{h X_{i}}{1+a\left(X_{u}+X_{i}\right)} P, \\
\frac{d P}{d t}=\frac{\gamma h\left(X_{u}+X_{i}\right)}{1+a\left(X_{u}+X_{i}\right)} P-e P .
\end{gathered}
$$

In this paper, if we further assume that the number of total host population keeps unchanged, that is, $K$ is a constant [ 25 , $35]$, then from assumption (7), the total vector population $X$ without $P$ can be governed by the logistic equation $d X / d t=$ $r X(1-X / M)$. Using assumptions that $S+I=K, b_{1}(K)=b_{1} K$, $K$ is a constant, and $d X / d t=r X(1-X / M)$, then (1) can be reduced as

$$
\begin{aligned}
& \frac{d I}{d t}=b_{1} \beta X_{i}(K-I)-(\mu+d) I, \\
& \frac{d X}{d t}=r X\left(1-\frac{X}{M}\right)-\frac{h X}{1+a X} P,
\end{aligned}
$$

$$
\begin{gathered}
\frac{d X_{i}}{d t}=b_{1} \alpha\left(X-X_{i}\right) I-w X_{i}-\frac{r X_{i}}{M} X-\frac{h X_{i}}{1+a X} P, \\
\frac{d P}{d t}=\frac{\gamma h X}{1+a X} P-e P,
\end{gathered}
$$

with initial conditions

$$
\begin{gathered}
\psi=\left\{\left(I(0), X(0), X_{i}(0), P(0)\right) \mid 0 \leq I(0) \leq K,\right. \\
\left.0 \leq X_{i}(0) \leq X(0) \leq M, P(0) \geq 0\right\} .
\end{gathered}
$$

It easily follows that model (2) can be studied in the positively invariant set:

$$
\begin{gathered}
\Omega=\left\{\left(I, X, X_{i}, P\right) \in R_{4}^{+} \mid 0 \leq I \leq K,\right. \\
\left.0 \leq X_{i} \leq X \leq M, P \geq 0\right\} .
\end{gathered}
$$

Without considering the predator, model (2) can be reduced to the following system:

$$
\begin{gathered}
\frac{d I}{d t}=b_{1} \beta X_{i}(K-I)-(\mu+d) I, \\
\frac{d X}{d t}=r X\left(1-\frac{X}{M}\right), \\
\frac{d X_{i}}{d t}=b_{1} \alpha\left(X-X_{i}\right) I-w X_{i}-\frac{r X_{i}}{M} X,
\end{gathered}
$$

and the positively invariant set of system (5) is

$$
T=\left\{\left(I, X, X_{i}\right) \in R_{3}^{+} \mid 0 \leq I \leq K, 0 \leq X_{i} \leq X \leq M\right\} .
$$

Compared with model (2.1) in [25], the main differences in our model are that (i) we assume that the vector population dynamics is governed by the logistic equation because the total host population on which vectors depend is a constant. (ii) Holling type II functional responses are used in our paper to describe the interaction between vectors and predators, which is more logistic and realistic because Holling type II 
functional responses introduce satiety effect in the predator population $[38,39]$. (iii) The linear per capita rate of contacts on hosts by vectors is considered.

The purpose of this paper is to investigate the effect of predators on the prevalence of disease among hosts by directly reducing the vector density. For this purpose, as preliminaries, we first give the equilibria and the reproduction numbers of (2) and (5) in the next section.

\section{The Equilibria and the Reproduction Number}

Lemma 1. The equilibria of system (2) are as follows.

(i) If the equilibrium value of the predator is zero, then there exist two disease-free equilibria, $E_{0}(0,0,0,0)$ and $E_{1}(0, M, 0,0)$, which always exist; equilibrium $E_{0}$ is the state of equilibrium of the uninfected host population only, while for equilibrium $E_{1}$, it is the equilibria of the uninfected hosts and vectors only and a disease equilibrium $E_{2}\left(I^{*}, M, X_{i}^{*}, 0\right)$ which exists if the threshold $R_{01}=\sqrt{b_{1}^{2} \alpha \beta K M /(b(\mu+d))}>1$.

(ii) If the equilibrium value of the predator is larger than zero, then there exists a disease-free equilibrium $E_{3}(0, \widehat{X}, 0, \widehat{P})$ if the threshold $R_{1}=\gamma h M /(e(1+$ $a M))>1$; equilibrium $E_{3}$ is the vector and predator equilibria in the absence of the pathogen and a disease equilibrium $E_{4}\left(\widehat{I}, \widehat{X}, \widehat{X}_{i}, \widehat{P}\right)$ if $R_{1}>1$ and $R_{02}=$ $\sqrt{b_{1}^{2} \alpha \beta K \widehat{X} /(b(\mu+d))}>1$, where

$$
\begin{gathered}
I^{*}=\frac{b_{1}^{2} \alpha \beta M K-b(\mu+d)}{b_{1}^{2} \alpha \beta M+b_{1} \alpha(\mu+d)}, \quad X_{i}^{*}=\frac{(\mu+d) I^{*}}{b_{1} \beta\left(K-I^{*}\right)}, \\
\widehat{X}=\frac{e}{\gamma h-a e}, \quad \widehat{P}=\frac{r}{h}(1+a \widehat{X})\left(1-\frac{\widehat{X}}{M}\right), \\
\widehat{I}=\frac{b_{1}^{2} \alpha \beta K \widehat{X}-b(\mu+d)}{b_{1}^{2} \alpha \beta \widehat{X}+b_{1} \alpha(\mu+d)}, \quad \widehat{X}_{i}=\frac{(\mu+d) \widehat{I}}{b_{1} \beta(K-\widehat{I})} .
\end{gathered}
$$

Inequality $R_{1}>1$ aims to ensure $\widehat{P}>0$, and by the next generation matrix, we find that

$$
\begin{gathered}
R_{01}=\sqrt{\frac{b_{1}^{2} \alpha \beta K M}{b(\mu+d)}}=\sqrt{K \frac{b_{1} \alpha}{b} \cdot M \frac{b_{1} \beta}{(\mu+d)}}, \\
R_{02}=\sqrt{\frac{b_{1}^{2} \alpha \beta K \widehat{X}}{b(\mu+d)}}=\sqrt{K \frac{b_{1} \alpha}{b} \cdot \widehat{X} \frac{b_{1} \beta}{(\mu+d)}}
\end{gathered}
$$

are the basic reproduction number of systems (5) and (2), respectively.

The biological meaning of $R_{01}$ and $R_{02}$ can be interpreted as follows. For $R_{01}$, since the predator of vectors is not introduced (i.e., $P=0$ ), then the total number of vector populations achieves the stable state $M$ and every vector is the susceptible and the number of the new infected vectors produced by each infected host over his/her expected infectious period is $M$. $\left(b_{1} \beta /(\mu+d)\right)$, and when the total number of host population reaches stable state $K$ and everyone is the susceptible, the number of new infected hosts produced by each infected vector (though the bite of the infected vector) over its expected infectious period is $K \cdot\left(b_{1} \alpha / b\right)$. The square root arises from the two "generations" required for an infected host or vector to "reproduce" itself.

While for the biological meaning of $R_{02}$, since the predator of vectors is introduced (that is $P>0$ ), then the total number of vector population achieves the stable state $\widehat{X}=e /(\gamma h-$ $a e)$; therefore, the number of the new infected vectors produced by each infected host over his/her expected infectious period is $\widehat{X} \cdot\left(b_{1} \beta /(\mu+d)\right)$, and when the total number of host populations reaches stable state $K$ and everyone is the susceptible, the number of new infected hosts produced by each infected vector (though the bite of the infected vector) over its expected infectious period is $K \cdot\left(b_{1} \alpha / b\right)$. The square root arises from the two "generations" required for an infected host or vector to "reproduce" itself.

In fact, by [40, 41], the basic reproduction number of (2) is expressed as

$$
R_{0}=\sqrt{\frac{b_{1}^{2} \alpha \beta K X}{(\mu+d)(w+(r / M) X+h P /(1+a X))}},
$$

where $X$ and $P$ are the disease-free equilibrium values. From the disease-free equilibrium $E_{3}(0, \widehat{X}, 0, \widehat{P})$, substitute $X=$ $\widehat{X}, P=\widehat{P}$ into (9), and denote $R_{0}$ as $R_{02}$ yields $R_{02}=$ $\sqrt{b_{1}^{2} \alpha \beta K \widehat{X} /(b(\mu+d))}$, which is the basic reproduction number of system (2).

In particular, if $P=0$, then from the disease-free equilibrium $E_{1}(0, M, 0,0)$, substitute $X=M, P=0$ into (9), and denote $R_{0}$ as $R_{01}$ yields $R_{01}=\sqrt{b_{1}^{2} \alpha \beta K M /(b(\mu+d))}$, which is the basic reproduction number of system (5).

Remark 2. Take $R_{0}$ as a function of $X(0<X \leq M)$; then from (9) $R_{01}>R_{02}$ if $R_{1}>1$ (i.e., $M>\widehat{X}$ ). That is, predators result into a reduction in $R_{0}$.

In particular, we obtain the following three possible equilibria of (5).

Lemma 3. There exist three possible equilibria for system (5), they are $E_{0}(0,0,0), E_{1}=(0, M, 0)$, which always exist, and $E_{2}=\left(I^{*}, M, X_{i}^{*}\right)$ which exists when $R_{01}>1$, where $I^{*}$ and $X_{i}^{*}$ are given in Lemma 1.

From (9), by adding the predator's attack rate $h$, then $R_{0}$ decreases and an increase in predation strength causes a decrease in the equilibrium proportion of infected hosts and vectors.

Now, the aim of biological control is to reduce the risk of disease outbreaks in the host population by introducing predators of the vector population. In other words, we need to find the critical condition or the critical values such as predator's attack rate $h$ to make $I(t) \rightarrow 0, X_{i}(t) \rightarrow 0$, as $t \rightarrow+\infty$. For this purpose, we need to study the dynamics of models (2) and (5). 


\section{Local Stability of the Equilibria for System (2)}

For analyzing the effect of predation on the prevalence of vector-transmitted disease, we first analyze the local stability of model (2).

Theorem 4. The local stability of equilibria for system (2) is as follows.

(i) The disease-free equilibrium $E_{0}$ (uninfected host population only) is unstable.

(ii) The disease-free equilibrium $E_{1}$ (only uninfected hosts and vectors) is locally asymptotically stable if $R_{01}<1$ and $R_{1}<1$.

(iii) The disease equilibrium $E_{2}$ (without predators) is locally asymptotically stable if $R_{01}>1$ and $R_{1}<1$.

(iv) The disease-free equilibrium $E_{3}$ (the vector and predator equilibria in absence of the pathogen) is locally asymptotically stable if $R_{02}<1$ and $1<R_{1}<1+$ $\gamma h /(a e(1+a M))$.

(v) The disease equilibrium $E_{4}$ (with predators) is locally asymptotically stable if $R_{02}>1$ and $1<R_{1}<1+$ $\gamma h /(a e(1+a M))$.

Proof. (i) Linearizing around the disease-free equilibrium $E_{0}$ (uninfected host population only), there exist four eigenvalues, they are $\lambda_{1}=-(\mu+d)<0, \lambda_{2}=r>0, \lambda_{3}=-w<0$, $\lambda_{4}=-e<0$. Thus, $E_{0}$ is unstable.

(ii) Linearizing around the disease-free equilibrium $E_{1}$ (only uninfected hosts and vectors), two eigenvalues are obtained, they are $\lambda_{1}=-r<0, \lambda_{2}=(\gamma h M /(1+a M))-e<0$ if $R_{1}<1$. The other two roots are determined by the quadratic equation

$$
\lambda^{2}+(b+\mu+d) \lambda+b(\mu+d)-b_{1}^{2} \alpha \beta M K=0 .
$$

The roots of (10) have negative real parts if and only if its coefficients are positive. Coefficient $(b+\mu+d)$ is always positive, while for coefficient $b(\mu+d)-b_{1}^{2} \alpha \beta M K$, it is positive if and only if $R_{01}<1$. Therefore, disease-free equilibrium $E_{1}$ is locally asymptotically stable if $R_{01}<1$ and $R_{1}<1$.

(iii) For the disease equilibrium $E_{2}$ (without predators) by linearizing around it, we obtain two eigenvalues, they are $\lambda_{1}=-r<0$ and $\lambda_{2}=\gamma h M /(1+a M)-e<0$ if $R_{1}<1$. The other two roots are determined by the quadratic equation

$$
\lambda^{2}+p_{1} \lambda+q_{1}=0
$$

where $p_{1}=\mu+d+b+b_{1} \alpha I^{*}+b_{1} \beta X_{i}^{*}>0$,

$$
\begin{aligned}
q_{1}= & \left(\mu+d+b_{1} \beta X_{i}^{*}\right)\left(b+b_{1} \alpha I^{*}\right) \\
& -b_{1}^{2} \alpha \beta\left(M-X_{i}^{*}\right)\left(K-I^{*}\right) .
\end{aligned}
$$

Equation (12) has two characteristic roots with negative real parts if and only if $q_{1}>0$. From the equilibrium expression $I^{*}=\left(b_{1}^{2} \alpha \beta M K-b(\mu+d)\right) /\left(b_{1}^{2} \alpha \beta M+b_{1} \alpha(\mu+d)\right)$, we have

$$
b_{1}^{2} \alpha \beta M K=\left[b_{1}^{2} \alpha \beta M+b_{1} \alpha(\mu+d)\right] I^{*}+b(\mu+d) \text {. }
$$

Substitute (13) into (12) and reduce it; yield $q_{1}=b b_{1} \beta X_{i}^{*}+$ $b_{1}^{2} \alpha \beta K X_{i}^{*}>0$ if $R_{01}>1$, Therefore, the disease equilibrium $E_{2}$ is locally asymptotically stable for $R_{01}>1$ and $R_{1}<1$.

(iv) Linearizing system (2) about the disease-free equilibrium $E_{3}$ (the vector and predator equilibria in absence of the pathogen) gives the following characteristic equation corresponding to the Jacobi matrix:

$$
\left|\begin{array}{cccc}
\lambda+(\mu+d) & 0 & -b_{1} \beta K & 0 \\
0 & \lambda-r+\frac{2 r \widehat{X}}{M}+\frac{r(1-\widehat{X} / M)}{1+a \widehat{X}} & 0 & \frac{h \widehat{X}}{1+a \widehat{X}} \\
-b_{1} \alpha \widehat{X} & 0 & \lambda+b & 0 \\
0 & -\frac{\gamma r(1-\widehat{X} / M)}{1+a \widehat{X}} & 0 & \lambda
\end{array}\right|=0 .
$$

Expanding by the last column and by further simplifying, the above characteristic equation can be reduced to the following equation:

$$
\begin{gathered}
{\left[\lambda^{2}+(b+\mu+d) \lambda+b(\mu+d)-b_{1}^{2} \alpha \beta K \widehat{X}\right]} \\
\times\left[\lambda^{2}+\left(\frac{2 r \widehat{X}}{M}+\frac{r(1-\widehat{X} / M)}{1+a \widehat{X}}-r\right) \lambda\right. \\
\left.+\frac{\gamma r h \widehat{X}(1-\widehat{X} / M)}{(1+a \widehat{X})^{2}}\right]=0 .
\end{gathered}
$$

Thus, the eigenvalues can be obtained by solving

$$
\begin{gathered}
\lambda^{2}+(b+\mu+d) \lambda+b(\mu+d)-b_{1}^{2} \alpha \beta K \widehat{X}=0, \\
\lambda^{2}+\left(\frac{2 r \widehat{X}}{M}+\frac{r(1-\widehat{X} / M)}{1+a \widehat{X}}-r\right) \lambda \\
+\frac{\gamma r h \widehat{X}(1-\widehat{X} / M)}{(1+a \widehat{X})^{2}}=0 .
\end{gathered}
$$

The roots of (16) have negative real parts if and only if $R_{02}<1$. On the other hand, (17) has two roots with negative real parts if and only if its coefficients are positive. Obviously, coefficient $\gamma r h \widehat{X}(1-\widehat{X} / M) /(1+a \widehat{X})^{2}$ is always positive if $R_{1}>$ 1 , while for coefficient $(2 r \widehat{X} / M)+(r(1-(\widehat{X} / M)) /(1+a \widehat{X}))-r$, we derive that $2 r \widehat{X} / M+(r(1-(\widehat{X} / M)) /(1+a \widehat{X}))-r>0$ is equivalent to $R_{1}<1+\gamma h /(a e(1+a M))$ by some mathematical deductions. Therefore, the disease-free equilibrium $E_{3}$ (with predators) is locally asymptotically stable if $R_{02}<1$ and $1<$ $R_{1}<1+\gamma h /(a e(1+a M))$.

(v) Linearizing system (2) about the disease equilibrium $E_{4}$ (with predators) gives the following characteristic equation corresponding to the Jacobi matrix: 


$$
\left|\begin{array}{cccc}
\lambda+(\mu+d)+b_{1} \beta \widehat{X}_{i} & 0 & -b_{1} \beta(K-\widehat{I}) & 0 \\
0 & \lambda-r+\frac{2 r \widehat{X}}{M}+\frac{r(1-\widehat{X} / M)}{1+a \widehat{X}} & 0 & \frac{h \widehat{X}}{1+a \widehat{X}} \\
-b_{1} \alpha\left(\widehat{X}-\widehat{X}_{i}\right) & -b_{1} \alpha \widehat{I}+\frac{r \widehat{X}}{M}-\frac{a r(1-\widehat{X} / M) \widehat{X}_{i}}{1+a \widehat{X}} & \lambda+b+b_{1} \alpha \widehat{I} & \frac{h \widehat{X}_{i}}{1+a \widehat{X}} \\
0 & -\frac{\gamma r(1-\widehat{X} / M)}{1+a \widehat{X}} & 0 & \lambda
\end{array}\right|=0 .
$$

Expanding by the first column and by further simplifying, the above characteristic equation can be reduced to the following equation:

$$
\begin{gathered}
{\left[\left(\lambda+\mu+d+b_{1} \beta \widehat{X}_{i}\right)\left(\lambda+b+b_{1} \alpha \widehat{I}\right)\right.} \\
\left.-b_{1}^{2} \alpha \beta(K-\widehat{I})\left(\widehat{X}-\widehat{X}_{i}\right)\right] \\
\times\left[\lambda^{2}+\left(\frac{2 r \widehat{X}}{M}+\frac{r(1-\widehat{X} / M)}{1+a \widehat{X}}-r\right) \lambda\right. \\
\left.+\frac{\gamma r h \widehat{X}(1-\widehat{X} / M)}{(1+a \widehat{X})^{2}}\right]=0 .
\end{gathered}
$$

From the above analysis, the equation $\lambda^{2}+(2 r \widehat{X} / M+r(1-$ $\widehat{X} / M) /(1+a \widehat{X})-r) \lambda+\gamma r h \widehat{X}(1-\widehat{X} / M) /(1+a \widehat{X})^{2}=0$ have two negative real parts if and only if $1<R_{1}<1+\gamma h /(a e(1+a M))$. So, in order to determine the asymptotically stable $E_{4}$, we only need to analyze the following equation:

$$
\begin{gathered}
\left(\lambda+\mu+d+b_{1} \beta \widehat{X}_{i}\right)\left(\lambda+b+b_{1} \alpha \widehat{I}\right) \\
-b_{1}^{2} \alpha \beta(K-\widehat{I})\left(\widehat{X}-\widehat{X}_{i}\right)=0 .
\end{gathered}
$$

For simplicity, set

$$
\begin{gathered}
p_{2}=\left(b+\mu+d+b_{1} \alpha \widehat{I}+b_{1} \beta \widehat{X}_{i}\right), \\
q_{2}=\left(\mu+d+b_{1} \beta \widehat{X}_{i}\right)\left(b+b_{1} \alpha \widehat{I}\right)-b_{1}^{2} \alpha \beta(K-\widehat{I})\left(\widehat{X}-\widehat{X}_{i}\right) .
\end{gathered}
$$

Then, (20) is equivalent to the following equation:

$$
\lambda^{2}+p_{2} \lambda+q_{2}=0
$$

where $p_{2}=\left(b+\mu+d+b_{1} \alpha \widehat{I}+b_{1} \beta \widehat{X}_{i}\right)>0$ if $R_{02}>1$ and $R_{1}>1$. For obtaining two roots of quadratic equation (22) with negative real parts, the coefficient $q_{2}$ should satisfy $q_{2}>$ 0 :

$$
\begin{aligned}
q_{2}= & \left(\mu+d+b_{1} \beta \widehat{X}_{i}\right)\left(b+b_{1} \alpha \widehat{I}\right)-b_{1}^{2} \alpha \beta(K-\widehat{I})\left(\widehat{X}-\widehat{X}_{i}\right) \\
= & (\mu+d) b+(\mu+d) b_{1} \alpha \widehat{I} \\
& +b b_{1} \beta \widehat{X}_{i}+b_{1}^{2} \alpha \beta K \widehat{X}_{i}+b_{1}^{2} \alpha \beta \widehat{X} \widehat{I}-b_{1}^{2} \alpha \beta K \widehat{X} .
\end{aligned}
$$

By $\widehat{I}=\left(b_{1}^{2} \alpha \beta K \widehat{X}-b(\mu+d)\right) /\left(b_{1}^{2} \alpha \beta \widehat{X}+b_{1} \alpha(\mu+d)\right)$, we have

$$
b_{1}^{2} \alpha \beta M K=\left[b_{1}^{2} \alpha \beta \widehat{X}+b_{1} \alpha(\mu+d)\right] \widehat{I}+b(\mu+d) .
$$

Substitute (24) into (23) and yield $q_{2}=b b_{1} \beta \widehat{X}_{i}+$ $b_{1}^{2} \alpha \beta K \widehat{X}_{i}>0$. Therefore, the disease equilibrium $E_{4}$ (with predators) is locally asymptotically stable if $R_{02}>1$ and $1<R_{1}<1+\gamma h /(a e(1+a M))$. This completes the proof of Theorem 4 .

\section{Global Stability and Hopf Bifurcation of System (2)}

The purpose of this section is to discuss the global stability and other dynamics for system (2) and obtain the control condition under which diseases can be controlled by predators preying on vectors. First, we give the following theorem.

Theorem 5. If the equilibrium value of the predator is zero, then for the disease-free equilibria $E_{1}(0, M, 0,0)$ and disease equilibrium $E_{2}\left(I^{*}, M, X_{i}^{*}, 0\right)$ (without predators) of system (2), the following properties hold.

(i) If $R_{01} \leq 1$ and $R_{1}<1$, then $\lim _{t \rightarrow+\infty}\left(I, X, X_{i}, P\right)=$ $E_{1}$.

(ii) If $R_{01}>1$ and $R_{1}<1$, then $\lim _{t \rightarrow+\infty}\left(I, X, X_{i}, P\right)=$ $E_{2}$.

Proof. (i) From the second equation of system (2), $d X / d t \leq$ $r X(1-X / M)$ and then $\lim _{t \rightarrow+\infty} \sup X(t) \leq M$. Since $R_{1}<1$, we consider $\varepsilon$, such that $\gamma h(M+\varepsilon) /(e(1+a(M+\varepsilon)))<1$ and $\gamma h(M+\varepsilon) /(1+a(M+\varepsilon))-e<0$. There exists $T>0$, such that $X(t) \leq M+\varepsilon$ for $t>T$. Therefore, $\dot{P} \leq(\gamma h(M+$ $\varepsilon) /(1+a(M+\varepsilon))-e) P$, and $\lim _{t \rightarrow+\infty} P(t)=0$, if $R_{1}<1$. So $\lim _{t \rightarrow+\infty} X(t)=M$. Thus, the limit system of (2) can be reduced to

$$
\begin{gathered}
\frac{d I}{d t}=b_{1} \beta X_{i}(K-I)-(\mu+d) I, \\
\frac{d X_{i}}{d t}=b_{1} \alpha I\left(M-X_{i}\right)-b X_{i} .
\end{gathered}
$$


Consider the Lyapunov function $L=b_{1} \beta K X_{i}+b I$. Its derivative along the solution of (25) is

$$
\begin{aligned}
L^{\prime}= & b_{1} \beta K\left[b_{1} \alpha I\left(M-X_{i}\right)-b X_{i}\right] \\
& +b\left[b_{1} \beta X_{i}(K-I)-(\mu+d) I\right] \\
= & I\left[b_{1}^{2} \alpha \beta K\left(M-X_{i}\right)-b b_{1} \beta X_{i}-b(\mu+d)\right] \\
\leq & \frac{I}{b(\mu+d)}\left[\frac{b_{1}^{2} \alpha \beta K M}{b(\mu+d)}-1\right] \\
= & \frac{I}{b(\mu+d)}\left[R_{01}^{2}-1\right] .
\end{aligned}
$$

Obviously, $L^{\prime} \leq 0$ if $R_{01} \leq 1$. Moreover, the equality $L^{\prime}=0$ holds if and only if $I=X_{i}=0$. That is, the maximal compact invariant set in $\left\{I(t), X(t) \in T: L^{\prime}=0\right\}$ is $\{E(0,0)\}$. By Lyapunov-LaSalle theorem [42], the equilibrium $E(0,0)$ is globally stable if $R_{01} \leq 1$; therefore $\lim _{t \rightarrow+\infty}\left(I, X, X_{i}, P\right)=E_{1}$ when $R_{01} \leq 1$ and $R_{1}<1$.

(ii) From the Jacobin of (25) we can verify that system (25) is a cooperative irreducible system in $R_{2}^{+}$[43]. By the Jacobian of (25), it follows that if $R_{01}<1$ the origin of system (25) is locally asymptotically stable and if $R_{01}>1$ the origin of system (25) is unstable. Following Smith [44], system (25) is strongly concave. Then it follows that if $R_{01}>1$ system (25) has an equilibrium $E\left(I^{*}, X_{i}^{*}\right)$, which is globally asymptotically stable. Since system (25) is the limiting system of (2), it follows from Theorem 2.3 in [45] that the unique positive equilibrium $E_{2}\left(I^{*}, M, X_{i}^{*}, 0\right)$ is a globally asymptotically stable equilibrium of system (2).

This completes the proof of Theorem 5 .

Based on Theorems 4 and 5, we have the following corollary for system (5), which is a special case of system (2) (without predators).

Corollary 6. For system (5), the following results hold.

(i) The disease-free equilibrium $E_{0}$ is a saddle-node with $x$-axis as its unstable manifold and $I-X_{i}$ plane as its stable manifold.

(ii) If $R_{01} \leq 1$, then $\lim _{t \rightarrow+\infty}\left(I, X, X_{i}\right)=E_{1}$, in which case, the vector-transmitted disease will eventually die out.

(iii) If $R_{01}>1$, then $\lim _{t \rightarrow+\infty}\left(I, X, X_{i}\right)=E_{2}$, in which case, the vector-transmitted disease will persist.

Remark 7. By Corollary 6, we see the disease will persist (stability of $E_{2}$ ) or die out (stability of $E_{1}$ ) in absent of vector predators. Such results fully depend on the basic reproduction number $R_{01}$ of system (5) which is bigger or smaller than one.

For system (2), there exist two important equilibria: the disease-free boundary equilibrium $E_{3}(0, \widehat{X}, 0, \widehat{P})$ and the disease equilibrium $E_{4}(\widehat{I}, \widehat{X}, \widehat{X}, \widehat{P})$ when the equilibrium value of the predator $(P)$ is larger than zero. With the help of the dynamics of these two equilibria, we can judge whether the disease can be eradicated in presence of the vector predators. For this purpose, we give the following theorem.

Theorem 8. Assume that the interior equilibrium $E_{2}\left(I^{*}, X^{*}\right.$, $\left.X_{i}^{*}\right)$ of the reduced system (5) exists and is globally stable $\left(R_{01}>\right.$ $1)$; then we have

(i) if $R_{02} \leq 1$ and $1<R_{1} \leq 1+\gamma h /(a e(1+a M))$, then $\lim _{t \rightarrow+\infty}\left(I, X, X_{i}, P\right)=E_{3}$, in which case, the infected hosts and the infected vectors will die out. Therefore, the vector-transmitted disease can be eradicated in presence of vector predators.

(ii) if $R_{02}>1$ and $1<R_{1} \leq 1+\gamma h /(a e(1+a M))$, then $\lim _{t \rightarrow+\infty}\left(I, X, X_{i}, P\right)=E_{4}$, in which case, the infected hosts and the infected vectors will persist. Therefore, the vector-transmitted disease will persist though in presence of vector predators.

(iii) if $R_{02}<1$ and $R_{1}>1+\gamma h /(a e(1+a M))$, then there exists a stable limit cycle in $(X, P)$ plane for system (2), in which case, despite these oscillations, predation can still eliminate the virus from the system, while vector densities are greater than zero.

Before giving the main proof, we need the following lemma.

Let $I=X_{i}=0$ in system (5) (without considering disease among hosts); then we have the following predatorprey model:

$$
\begin{gathered}
\frac{d X}{d t}=r\left(1-\frac{X}{M}\right) X-\frac{h X}{1+a X} P, \\
\frac{d P}{d t}=\frac{\gamma h X}{1+a X} P-e P .
\end{gathered}
$$

Lemma 9 (see [46]). For system (27), the following results hold.

(i) There exists the unique positive equilibrium $(\widehat{X}, \widehat{P})=$ $(e /(\gamma h-a e),(r / h)(1+a \widehat{X})(1-\widehat{X} / M))$ if $R_{1}>1$.

(ii) The positive equilibrium $(\widehat{X}, \widehat{P})$ is a globally asymptotically focus if $1<R_{1} \leq 1+\gamma h /(a e(1+a M))$ and when $R_{1}>1+\gamma h /(a e(1+a M)),(\widehat{X}, \widehat{P})$ is an unstable focus; moreover, there exists a stable limit cycle for (27). This phenomenon corresponds to a supercritical Hopf bifurcation.

System (27) is a classical predator-prey model and some authors have studies on the dynamics of (27) or models similar to (27). Here, we take the attack rate $h$ as bifurcation parameter and give the proof for the occurrence of the bifurcation when $h=\widetilde{h}=e(1+a M) /(\gamma(M-1 / a))$, where $\tilde{h}$ satisfies equation $R_{1}=1+\gamma h /(a e(1+a M))$.

Proof. For equation (27), there exists the unique positive equilibrium $(\widehat{X}, \widehat{P})$ if $R_{1}>1$. It is easy to get that characteristic equation of $(27)$ at $(\widehat{X}, \widehat{P})$ is

$$
\lambda^{2}+p \lambda+q=0
$$


where $p=\left(-r+2 r \widehat{X} / M+h \widehat{P} /(1+a \widehat{X})^{2}\right), q=(\gamma h \widehat{P} /(1+$ $\left.a \widehat{X})^{2}\right)(h \widehat{X} / 1+a \widehat{X})$.

Obviously, the above characteristic equation has only two roots and they can be expressed as

$$
\lambda_{1,2}=\frac{-p \pm \sqrt{p^{2}-4 q}}{2} .
$$

Note that $q>0$. Consequently, when $p>0$, that is, $R_{1}<1+\gamma h /(a e(1+a M)$ ) (which can be obtained by some mathematical deduction) or $h<\widetilde{h}$, the above two characteristic roots have negative real parts and the interior equilibrium $(\widehat{X}, \widehat{P})$ is asymptotically stable. Therefore, $h=\widetilde{h}$ is a bifurcation point of (27) about the interior equilibrium $(\widehat{X}, \widehat{P})$.

For characteristic equation $\lambda^{2}+p \lambda+q=0$, let $\lambda=\alpha(h)+$ $i w(h)$; then there exists a pair of purely imaginary roots $\pm w i=$ $\pm \sqrt{q} i$ when $p=0$ (i.e., $h=\widetilde{h}$ ). Therefore, $\alpha(\widetilde{h})=0, w(\widetilde{h})>0$. Differentiating $\lambda^{2}+p \lambda+q=0$ with respect to $h$, we have

$$
\frac{d \lambda(h)}{d h}=-\frac{1}{2 \lambda(h)+p(h)}\left(\lambda(h) \cdot \frac{d p(h)}{d h}+\frac{d q(h)}{d h}\right),
$$

and noticing the fact that $p(\widetilde{h})=-2 \alpha(\widetilde{h})=0$ and $\lambda= \pm w i=$ $\pm \sqrt{q} i$ when $h=\widetilde{h}$, so

$$
\left.\frac{d \lambda(h)}{d h}\right|_{h=\tilde{h}}=-\frac{1}{2 w i}\left(\left.w i \cdot \frac{d p(h)}{d h}\right|_{h=\tilde{h}}+\left.\frac{d q(h)}{d h}\right|_{h=\tilde{h}}\right),
$$

then

$$
\begin{aligned}
\left.\frac{d \alpha(h)}{d h}\right|_{h=\widetilde{h}}= & -\left.\frac{1}{2} \frac{d p(h)}{d h}\right|_{h=\widetilde{h}} \\
= & -\frac{1}{2} \frac{d\left(-r+2 r \widehat{X} / M+h \widehat{P} /(1+a \widehat{X})^{2}\right)}{d h} \mid h=\widetilde{h} \\
= & \frac{1}{2} \frac{e \gamma}{(\gamma h-a e)^{2}} \\
& \times\left[\left(\left(2(1+a \widehat{X})^{2}-r(1+a \widehat{X})\right.\right.\right. \\
& \left.\quad+r a M\left(1-\frac{\widehat{X}}{M}\right)\right) \\
& \left.\left.\times\left(M(1+a \widehat{X})^{2}\right)^{-1}\right)\right] \mid h=\widetilde{h} .
\end{aligned}
$$

When $h=\widetilde{h}$, we have $p(\widetilde{h})=0$. On the other hand, by mathematical deducing, we obtain that $p(\widetilde{h})=0$ is equivalent to $\widehat{X}=(a M-1) /(2 a)$. Substitute $\widehat{X}=(a M-1) /(2 a)$ into the above equation; it follows that

$$
\left.\frac{d \alpha(h)}{d h}\right|_{h=\tilde{h}}=\frac{1}{4} \frac{e \gamma}{(\gamma h-a e)^{2}} \frac{(a M+1)^{2}}{M(1+a \widehat{X})^{2}}>0 .
$$

Thus, (27) will undergo a Hopf bifurcation about the interior equilibrium $(\widehat{X}, \widehat{P})$ as $h$ passes through the value $\widetilde{h}$ (i.e., $R_{1}=$ $1+\gamma h /(a e(1+a M)))$.

This completes the proof for the occurrence of the bifurcation.

Based on Lemma 9, we give the proof of Theorem 8.

Proof. (i-ii) By Lemma 9, the solutions $X(t)$ and $P(t)$ of system (2) satisfy $\lim _{t \rightarrow+\infty}(X(t), P(t))=(\widehat{X}, \widehat{P})$ if $1<R_{1} \leq$ $1+\gamma h /(a e(1+a M))$. Then the limit system of (2) is reduced to

$$
\begin{gathered}
\frac{d I}{d t}=b_{1} \beta(K-I) X_{i}-(\mu+d) I, \\
\frac{d X_{i}}{d t}=b_{1} \alpha I\left(\widehat{X}-X_{i}\right)-b X_{i} .
\end{gathered}
$$

Similar to the proof of Theorem 5, we can prove that $\lim _{t \rightarrow+\infty}\left(I, X_{u}, X_{i}, P\right)=E_{3}$ if $1<R_{1} \leq 1+\gamma h /(a e(1+a M))$ and $R_{02}<1$, and $\lim _{t \rightarrow+\infty}\left(I, X, X_{i}, P\right)=E_{4}$ if $R_{02}>1$ and $1<R_{1} \leq 1+\gamma h /(a e(1+a M))$.

(iii) From the characteristic equation (15) of equilibrium $E_{3}$ of system (2), we have $\lim _{t \rightarrow+\infty}\left(I(t), X_{i}(t)\right)=(0,0)$ if $R_{02}<1$. Then by Lemma 9 , then there exists a stable limit cycle of $(X, P)$ plane for system (2) if $R_{02}<1$ and $R_{1}>$ $1+\gamma h /(a e(1+a M))$.

This completes the proof of Theorem 8 .

Moreover, by simulation we obtain that if $R_{02}>1$ and $R_{1}>1+\gamma h /(a e(1+a M))$, then there exists a stable limit cycle for system (2), in which case predation cannot eliminate the virus from the system and eventually leads to stable oscillations (See Figure 5).

From Theorem 8, if $R_{02}>1$ and $R_{1}>1$, then $I(t) \rightarrow \widehat{I}$, $X_{i}(t) \rightarrow \widehat{X}_{i}$, as $t \rightarrow+\infty$; thus vector-borne diseases would persist though predators are introduced, while if $R_{02}<1$ and $R_{1}>1$, then $I(t) \rightarrow 0, X_{i}(t) \rightarrow 0$, as $t \rightarrow+\infty$, then vector-borne diseases will be extinct by introducing vector predators. Thus, we have the following corollary.

Corollary 10. Assume that the vector-borne disease persists without predators; in other words, the interior equilibrium $E_{3}\left(I^{*}, X_{u}^{*}, X_{i}^{*}\right)$ of the system (5) exists and is globally asymptotically stable $\left(R_{01}>1\right)$; then by introducing vector predators $\left(R_{1}>1\right.$ to ensure $\left.P>0\right)$, we have

(i) the vector-borne disease can beeliminated if $R_{02} \leq 1$;

(ii) the existing vector-borne disease will persist if $R_{02}>1$.

Remark 11. From Corollary 10, the persistent disease in absence of predators $\left(R_{01}>1\right)$ will tend to extinct if $R_{02}<1$ and will persist if $R_{02}>1$ by introducing vector predators $\left(R_{1}>1\right.$ to ensure $\left.P>0\right)$. Therefore, the reproduction number $R_{02}$ is the threshold value determining the persistence or extinction of the existing vector-borne disease.

If we choose predator's attack rate $h$ as parameter, then from $R_{02}=1$, we have $h=(e / \gamma)\left[a+b_{1}^{2} \alpha \beta K /(b(\mu+d))\right]$. Denote $h^{*}=(e / \gamma)\left[a+b_{1}^{2} \alpha \beta K /(b(\mu+d))\right]$ and then yield the following corollary. 


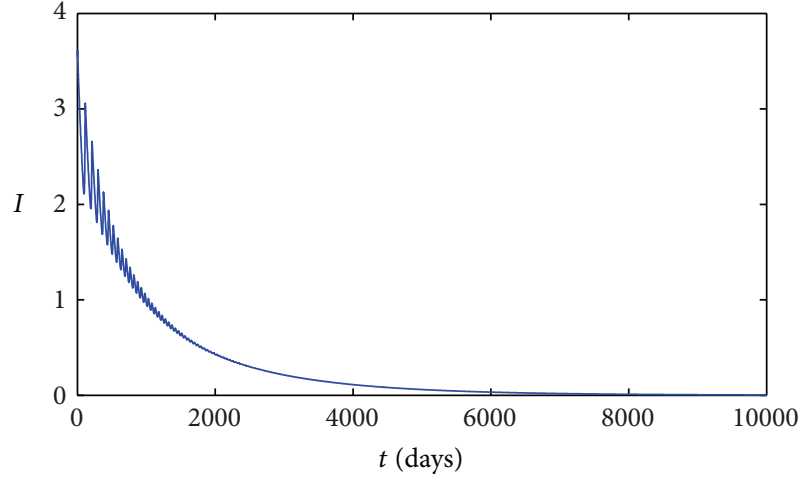

(a)

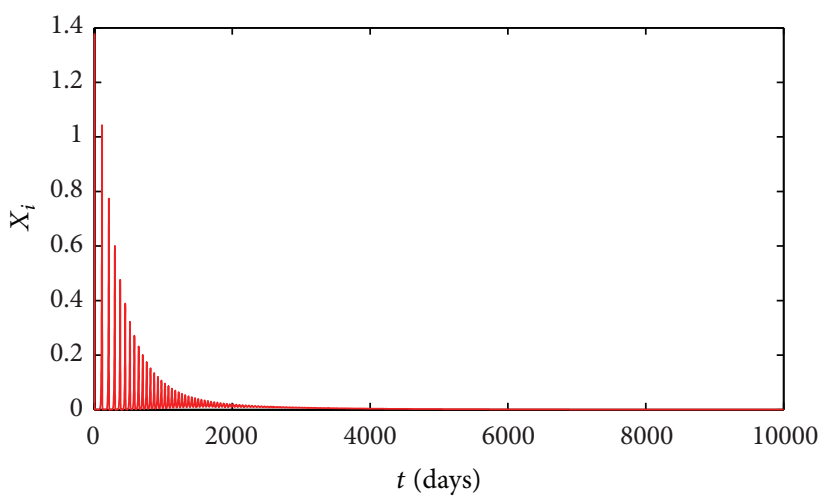

(c)

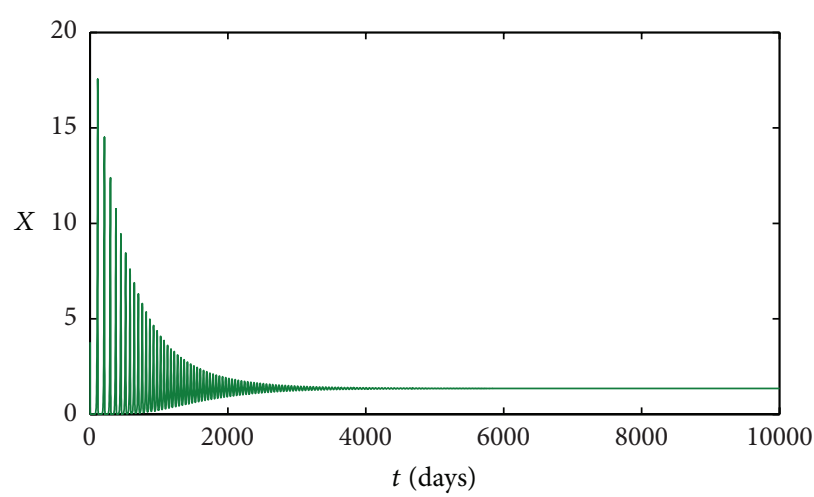

(b)

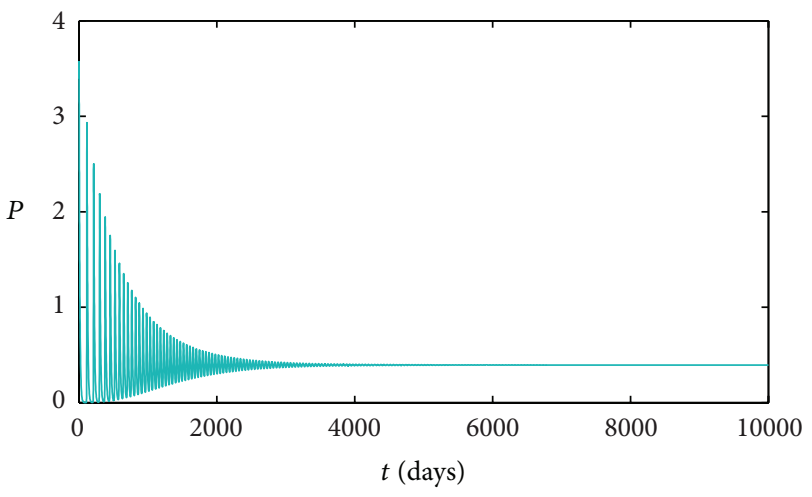

(d)

FiguRE 2: Numerical simulation for Case 1, with $a=0.01, h=0.5$, and other parameters fixed in Table 1 . The vector-borne disease tends to extinct in presence of vector predators.

Corollary 12. Assume that the vector-borne disease persists in absence of vector predator $\left(R_{01}>1\right)$; then by introducing vector predators,

(i) the vector-transmitted disease can be eliminated if predator's attack rate $h$ satisfies $h>h^{*}$;

(ii) the existing vector-borne disease will persist if predator's attack rate $h$ satisfies

$$
\frac{e}{\gamma}\left[a+\frac{1}{M}\right]<h<h^{*}
$$

Remark 13. Corollaries 10 and 12 give the conditions to judge when will the existing vector-borne disease be eradicated by introducing predators to reduce vector densities, which provides us with qualitative scheme for disease control.

Remark 14. Based on Corollary 10, other similar results can be derived if taking other predator parameters such as the conversation rate $\gamma$ as parameter. In this paper, we mainly investigate the persistence or extinction of disease under different attack rate $h$ of the predator population, especially in the following numerical simulations.

\section{Numerical Simulations}

In this section, we present some numerical simulations to support the analytical results for Cases 1, 2, and 3.
Case 1 (the effects of predators on the persistence or eradication of virus transmitted by arthropod vectors). Keep values of $\mu, d, b_{1}, \alpha, \beta, M, K, r, w, b$ unchanged in Table 1 . By simple calculations, the basic reproduction number $R_{01}=$ $5.7735>1$. By Corollary 6, without vector predators, the vector-transmitted disease will persist. In such a case, we introduce the predator. Take $a=0.01, h=0.5$ and keep values $\gamma$ and $e$ fixed in Table 1 and then by direct computation, we have $R_{02}=0.9492<1, R_{1}=25.00>1, R_{1}-1-\gamma h /($ ae $(1+$ $a M))=-26.00 \leq 0$; then by Theorem 8 , the disease-free equilibrium $E_{3}(0, \widehat{X}, 0, \widehat{P})$ is globally asymptotically stable, which indicates that the existing vector-transmitted disease will die out in presence of vector predators (see Figure 2).

Keep all the values unchanged in Figure 2 except that $h=0.2$, then by simple calculations, $R_{01}=5.7735>1$, $R_{02}=1.5162>1, R_{1}=10.00>1, R_{1}-1-\gamma h /(a e(1+a M))=$ $-11.00 \leq 0$. Therefore, by Theorem 8 , the disease equilibrium $E_{4}(\widehat{I}, \widehat{X}, \widehat{X}, \widehat{P})$ is globally asymptotically stable, which indicates that the vector-transmitted disease will be persistent though in presence of vector predators (see Figure 3).

Remark 15. By Figures 2 and 3, the disease will be persistent or tend to be extinct in presence of predators, which greatly depends on the predator strength. It can be easily verified that if the attack rate $h$ satisfies $h=0.2<h^{*}$, then by Corollary 12 the disease will persist. While if the attack rate $h$ is added up to 0.5 and satisfies $h=0.5>0.4511=h^{*}$, then by Corollary 12, 


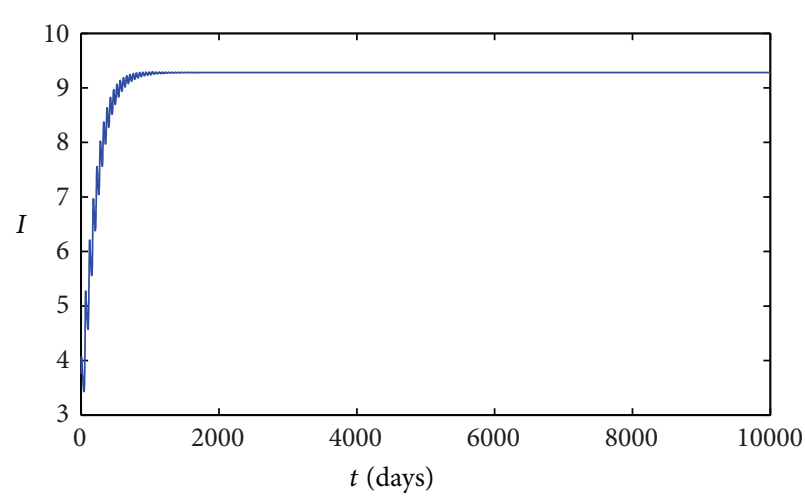

(a)

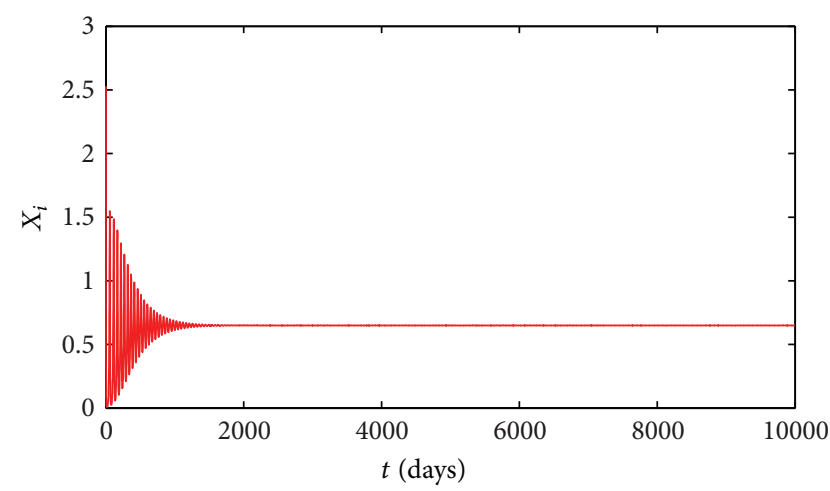

(c)

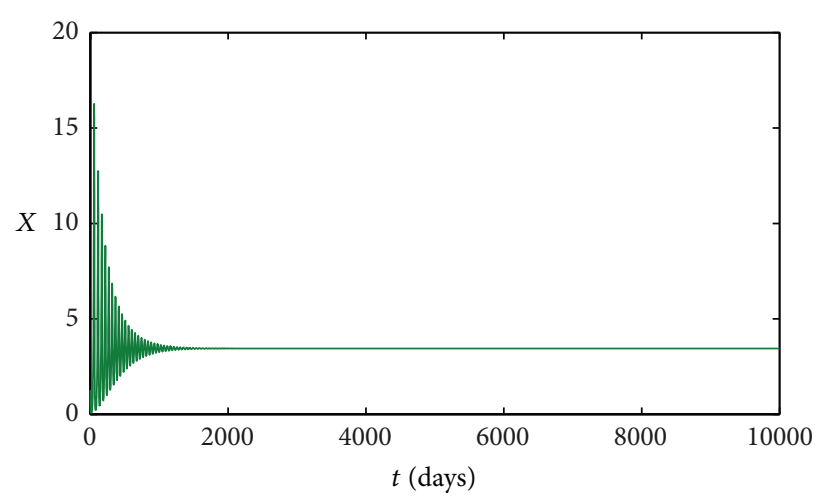

(b)

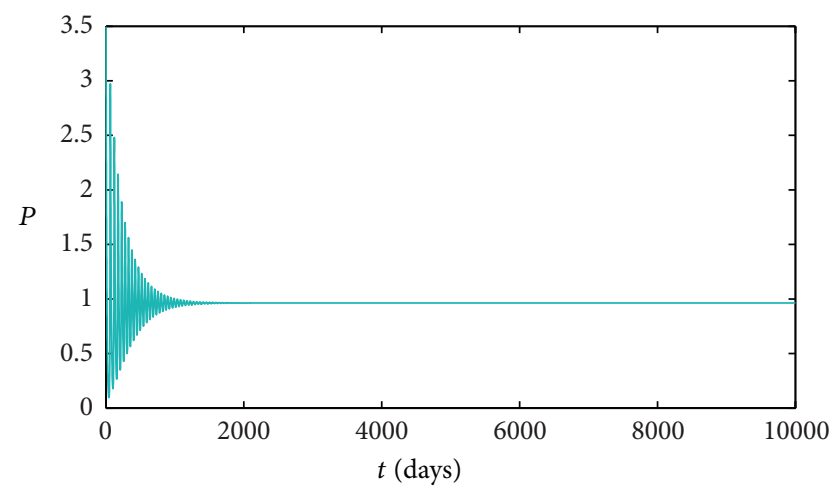

(d)

FIGURE 3: Numerical simulation for Case 1 , with $a=0.01, h=0.2$, and other parameters fixed in Table 1 . The vector-borne disease will persist though in presence of vector predators.

the disease dies out. Moreover, from Figure 2, by enhancing the attack rate $h$, the reduced vector densities rather than outright eradication can suffice to eradicate disease, which is different to predators used in pest control where predations aim to eradicate pests.

Case 2 (the existence of stable limit cycles). Keep values of $\mu, d, b_{1}, \alpha, \beta, M, K, r, w, b$ unchanged in Case 1 ; then $R_{01}=$ $5.7735>1$; that is, the disease persists without predator. Take $a=0.03, h=0.65$, and keep values $\gamma$ and $e$ unchanged in Case 1. By simple calculations, $R_{02}=0.8399<1, R_{1}=19.5>1$, $R_{1}-1-\gamma h /(a e(1+a M))=5.5>0$; then by Theorem 8 , there exists a stable limit cycle in $(X-P)$ plane for system (2), but the infected host and vector population level tend to zero (see Figure 4).

Keep all values of system (2) unchanged in Figure 4 except that $h=0.12$; then by simple calculations, $R_{01}=5.7735>1$, $R_{02}=2.1082>1, R_{1}=3.6>1, R_{1}-1-\gamma h /(a e(1+a M))=$ $0.2>0$. By numerical simulation, there exists a stable limit cycle for system (2) (see Figure 5).

Remark 16. From Figures 4 and 5, we can see that periodic solutions exist in presence of vector predators, suggesting the occurrence of supercritical Hopf bifurcation. From Figure 5, the infected hosts, the vectors including infected and susceptible ones, and the predators oscillate, leading to the persistence of the vector-transmitted disease though predators are introduced. By enhancing predators' attack rate $h$ such that $h=0.65>0.4644=h^{*}$, then by Corollary 12, we find that predation can lead to extinction of the disease, despite the oscillations of the predator and the vector (see Figure 4.).

Case 3 (the comparing outcomes between models with and without the predator population). Keep values of $\mu, d, b_{1}, \alpha, \beta, M, K, r, w, b$ unchanged in Case 1 ; then $R_{01}=$ $5.7735>1$; that is, the disease persists without predator. Take predator's attack rate $h$ as parameter and keep $\gamma, e$, and $c$ unchanged in Case 1; we study how different values of $h$ affect the disease prevalence. In absence of the predator, that is, $h=0$, then the disease persists (see the above curve of Figure 6(a)). By introducing predators of the vector population, we find that the equilibrium infection levels have been reduced and also the onset of an epidemic has been delayed if the predator attack rate $h=0.2$. However, the disease has not been eradicated (see the below curve of Figure 6(a)). By increasing the attack rate of predators $h$, such that $h=0.5$, then from the below curve of Figure 6(b), we can see that the vector-borne disease can be eradicated by introducing vector predators.

Remark 17. From Figure 6, we conclude that an increase in predator's attack rate can not only reduce equilibrium infection levels, but also delay the onset of an epidemic (see 

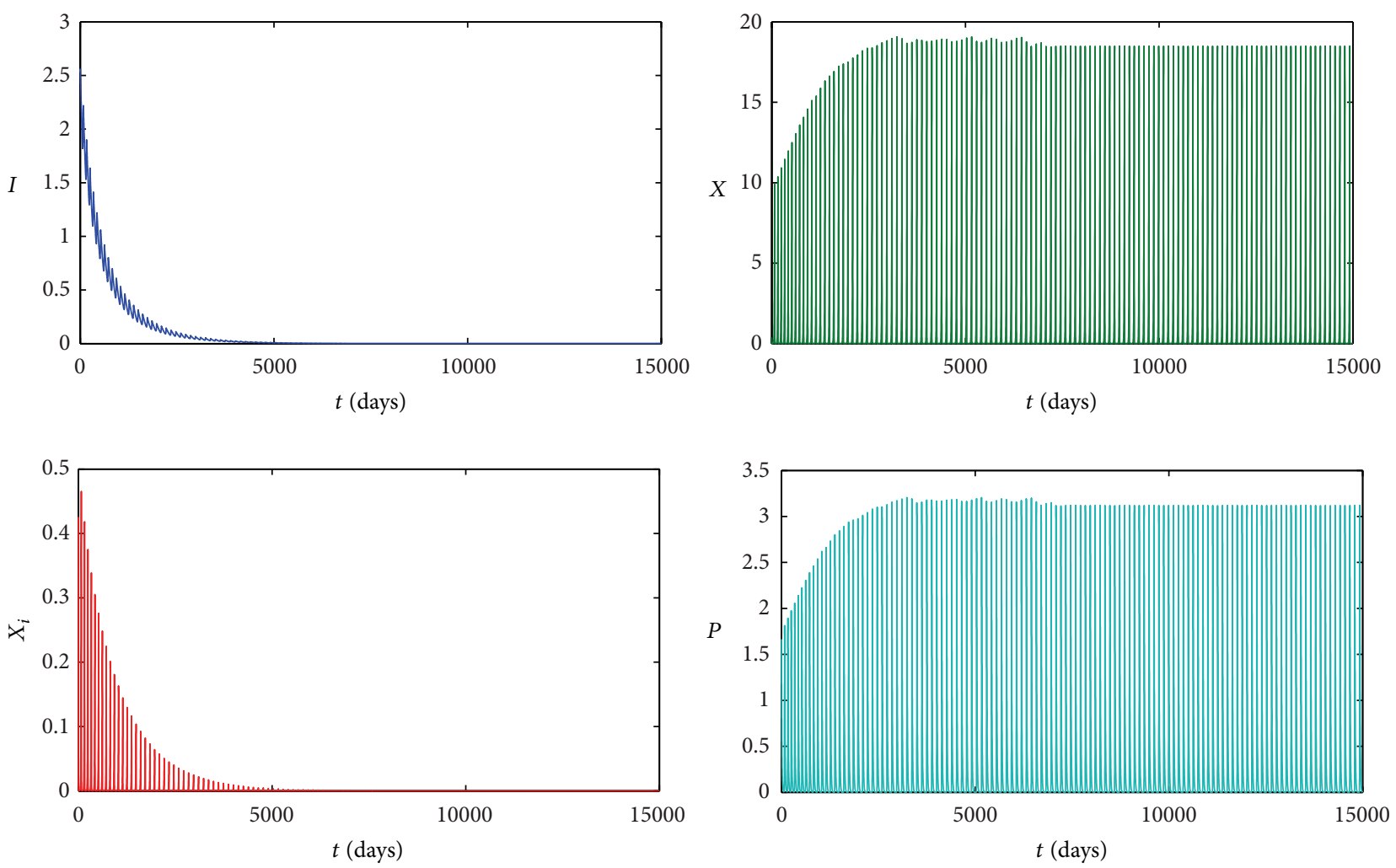

(a)

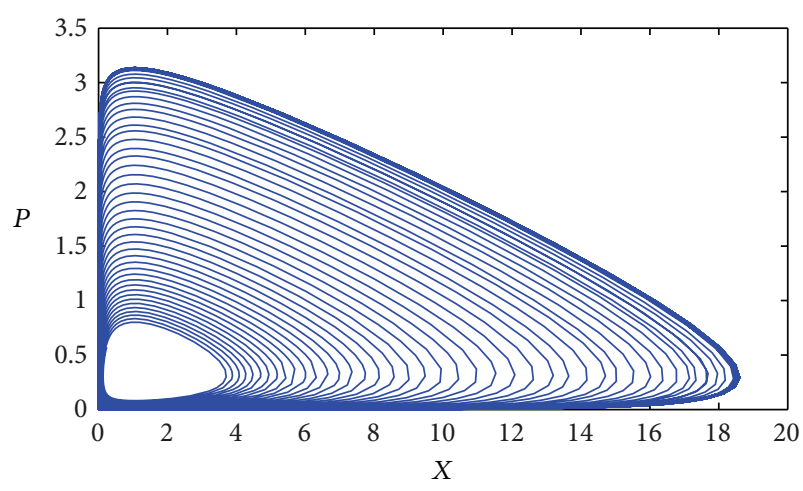

(b)

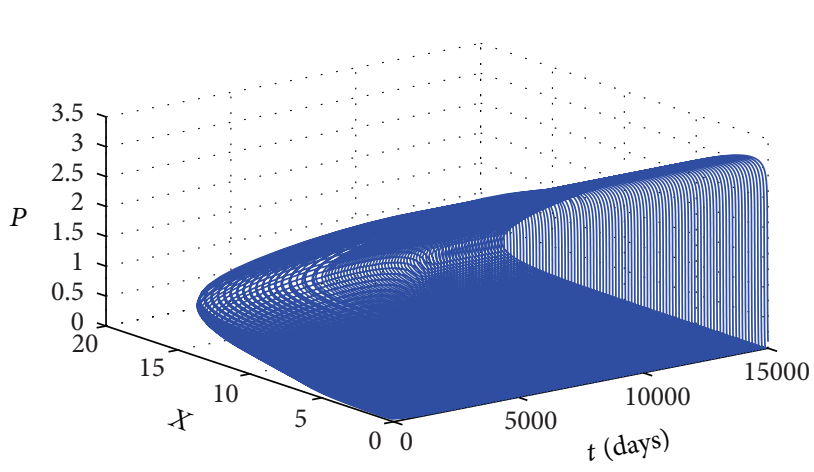

(c)

FIGURE 4: Numerical simulation for Case 2, with $a=0.03, h=0.65$, and other parameters fixed in Table 1. A stable limit cycle occurs in $X P$ plane of system (2). (a) Temporal plots for system variables. (b) Phase portrait in XP plane. (c) Phase portrait in $t X P$ space.

Figure 6(a)). Moreover, predation can eradicate virus if the attack rate $h$ satisfies $h>h^{*}$ (see Figure 6(b)).

\section{Concluding Remarks}

In this paper, we mainly propose a host, vector, and predator model to investigate the impact of the predator on the outbreak and extinction of vector-transmitted disease. Some sufficient conditions ensuring the globally asymptotically stability for the disease-free and disease equilibria are derived in absence and presence of predators. Moreover, complex dynamical phenomena such as Hopf bifurcation appear due to the stable focus losing stability. Taking the attack rate $h$ as the bifurcation parameter, we derive the threshold condition $h^{*}$ based on the stability results such that the vector-transmitted disease can be eradicated by introducing vector predators when $h>h^{*}$. Some numerical simulations are illustrated to support the theoretical analysis of research, also used for better comparison of the disease prevalence with or without predators. Moreover, since the viewpoint of pest control, multipredators have been testified more effectively on a single prey than one predator and another possible extension would be coupling our host-vector model with the prey and multipredator model studied in $[22,23]$. 


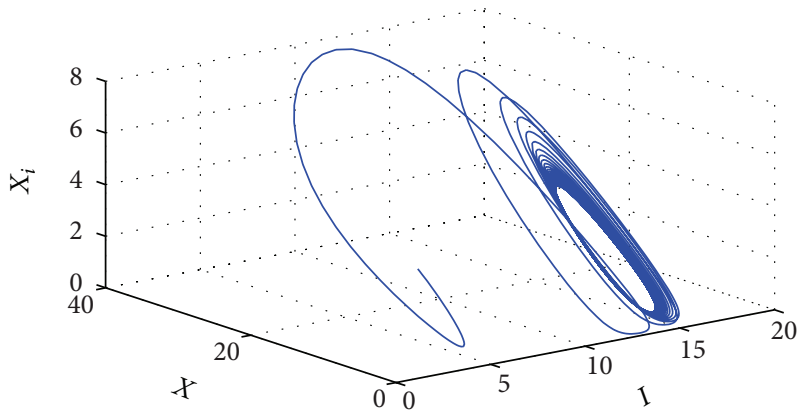

(a)

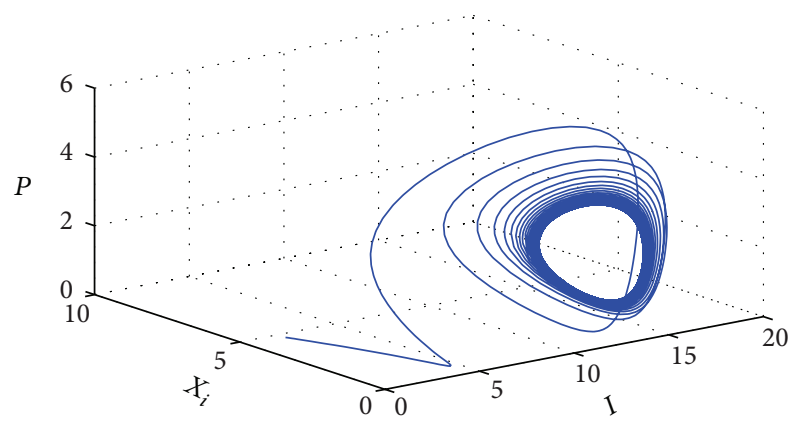

(c)

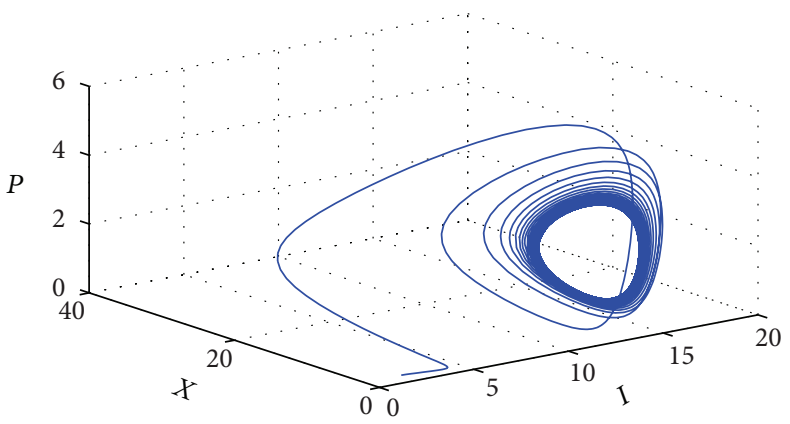

(b)

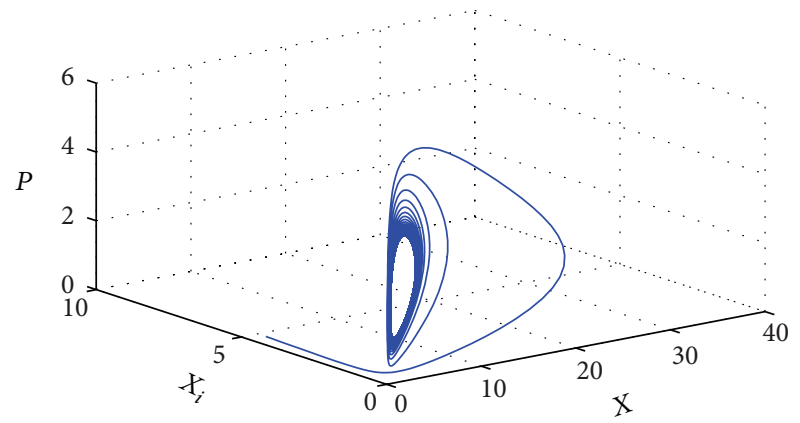

(d)

Figure 5: Numerical simulation for Case 2, with $a=0.03, h=0.12$, and other parameters fixed in Table 1. A stable limit cycle occurs for system (2).

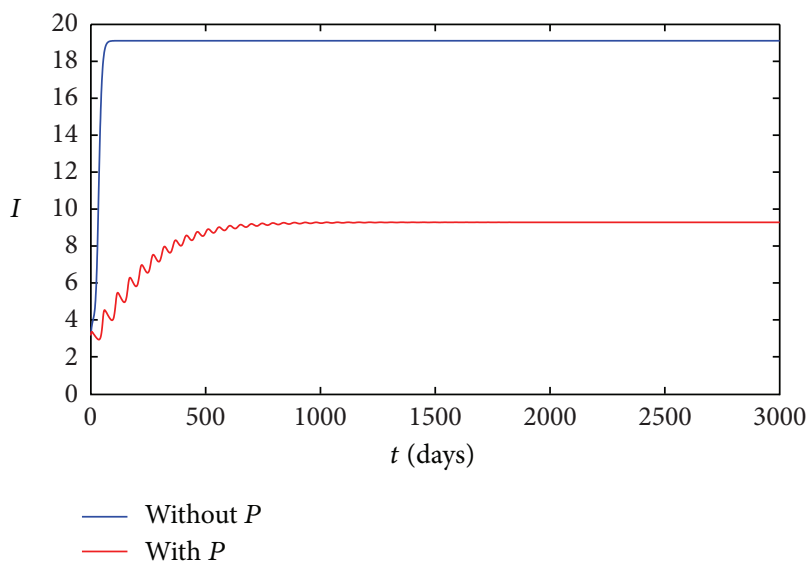

(a)

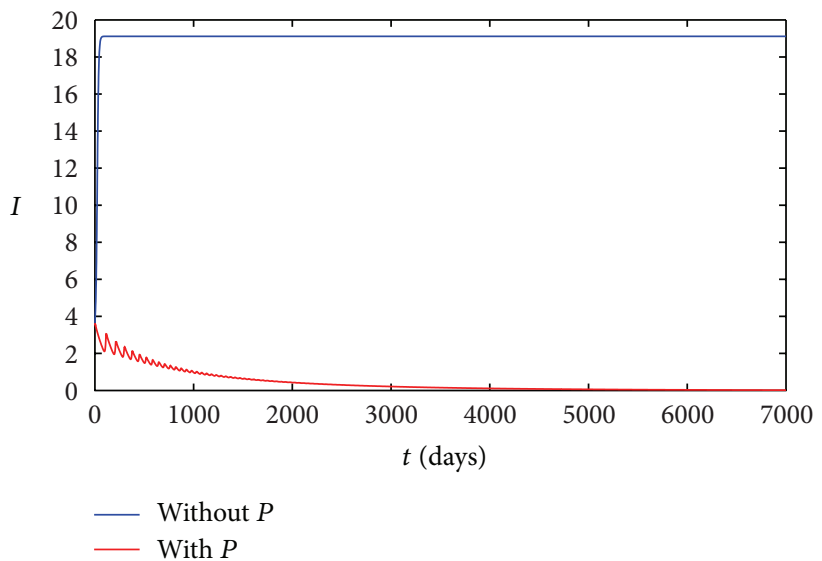

(b)

FIGURE 6: The population densities of the infected hosts with and without vector predators.

Therefore, we can further continue our work about the effect of multipredator on the prevalence of vector-borne disease.

\section{Conflict of Interests}

The authors declare that there is no conflict of interests regarding the publication of this paper.

\section{Acknowledgments}

The authors would like to thank the editor and the reviewers for their careful reading and valuable comments which greatly improve the presentation of the article. The work is supported by the National Natural Science Foundation of China (grant no. 70871056 and 71271103), the Six Talents Peak Foundation of Jiangsu Province, the National 
TABLE 1: Definition of parameters and values used in simulations (time unit is selected as day).

\begin{tabular}{|c|c|c|c|}
\hline Parameter & Description & Estimated value/range & Resource \\
\hline$\mu$ & Natural death rate of plants & 0.003 & {$[38]$} \\
\hline$d$ & Disease-induced mortality rate of plants & 0.003 & {$[38]$} \\
\hline$b_{1}$ & Per capita rate of contacts on plant hosts by vectors & 1 & Assumed \\
\hline$\alpha$ & $\begin{array}{l}\text { The disease transmission probability from infected plant hosts to uninfected vectors } \\
\text { (=the acquisition rate of virus) }\end{array}$ & 0.008 & {$[38]$} \\
\hline$\beta$ & $\begin{array}{l}\text { The disease transmission probability from infected vectors to uninfected plants } \\
\text { (=the inoculation rate of virus) }\end{array}$ & 0.008 & {$[38]$} \\
\hline$M$ & Vector carrying capacity & 50 & Assumed \\
\hline$K$ & Total plant population & 20 & Assumed \\
\hline$r$ & Intrinsic birth rate of vectors & 0.2 & {$[38]$} \\
\hline$w$ & Natural death rate of vectors & 0.12 & {$[38]$} \\
\hline$b$ & Natural birth rate of vectors & 0.32 & {$[38]$} \\
\hline$h$ & Prey searching rate & $0.1-0.65$ & Assumed \\
\hline$a$ & Predator's satiety rate & $0.01-0.03$ & Assumed \\
\hline$\gamma$ & Predator's conversation factor & 0.15 & {$[46]$} \\
\hline e & Predator's mortality rate & 0.1 & {$[46]$} \\
\hline
\end{tabular}

Research Subject of Education Information Technology (grant no. 126240641), and the Innovative Foundation for Doctoral Candidate of Jiangsu Province, China (grant no. CXZZ13_0687).

\section{References}

[1] N. T. J. Bailey, The Biomathematics of Malaria, Charles Griffin \& Company, High Wycombe, UK, 1982.

[2] J. Hemingway and H. Ranson, "Insecticide resistance in insect vectors of human disease," Annual Review of Entomology, vol. 45, pp. 371-391, 2000.

[3] K. F. Haynes, "Sublethal effects of neurotoxic insecticides on insect behavior," Annual Review of Entomology, vol. 33, pp. 149168,1988 .

[4] D. W. Jenkins, "Pathogens, parasites and predators of medically important arthropods. Annotated list and bibliography," Bulletin of the World Health Organization, vol. 30, pp. 1-150, 1964.

[5] E. Legner, "Biological control of diptera of medical and veterinary importance," Journal of Vector Ecology, vol. 20, pp. 59-120, 1995.

[6] J. R. Stauffer Jr., M. E. Arnegard, M. Cetron et al., "Controlling vectors and hosts of parasitic diseases using fishes," BioScience, vol. 47, no. 1, pp. 41-49, 1997.

[7] M. Samish and J. Rehacek, "Pathogens and predators of ticks and their potential in biological control," Annual Review of Entomology, vol. 44, pp. 159-182, 1999.

[8] E.-J. Scholte, K. Ng'Habi, J. Kihonda et al., "An entomopathogenic fungus for control of adult African malaria mosquitoes," Science, vol. 308, no. 5728, pp. 1641-1642, 2005.

[9] R. Kumar and J.-S. Hwang, "Larvicidal efficiency of aquatic predators: a perspective for mosquito biocontrol," Zoological Studies, vol. 45, no. 4, pp. 447-466, 2006.

[10] R. S. Ostfeld, A. Price, V. L. Hornbostel, M. A. Benjamin, and F. Keesing, "Controlling ticks and tick-borne zoonoses with biological and chemical agents," BioScience, vol. 56, no. 5, pp. 383-394, 2006.

[11] K. Walker and M. Lynch, "Contributions of Anopheles larval control to malaria suppression in tropical Africa: review of achievements and potential," Medical and Veterinary Entomology, vol. 21, no. 1, pp. 2-21, 2007.

[12] B. Kay and V. S. Nam, "New strategy against Aedes aegypti in Vietnam," The Lancet, vol. 365, no. 9459, pp. 613-617, 2005.

[13] P. Kittayapong, S. Yoksan, U. Chansang, C. Chansang, and A. Bhumiratana, "Suppression of dengue transmission by application of integrated vector control strategies at sero-positive GIS-based foci," The American Journal of Tropical Medicine and Hygiene, vol. 78, no. 1, pp. 70-76, 2008.

[14] S. K. Ghosh, S. N. Tiwari, T. S. Sathyanarayan et al., "Larvivorous fish in wells target the malaria vector sibling species of the Anopheles culicifacies complex in villages in Karnataka, India," Transactions of the Royal Society of Tropical Medicine \& Hygiene, vol. 99, no. 2, pp. 101-105, 2005.

[15] S. K. Ghosh and A. P. Dash, "Larvivorous fish against malaria vectors: a new outlook," Transactions of the Royal Society of Tropical Medicine \& Hygiene, vol. 101, no. 11, pp. 1063-1064, 2007.

[16] R. F. Luck, B. M. Shepard, and P. E. Kenmore, "Experimental methods for evaluating arthropod natural enemies," Annual Review of Entomology, vol. 33, pp. 367-389, 1988.

[17] G. Zehnder, G. M. Gurr, S. Kühne, M. R. Wade, S. D. Wratten, and E. Wyss, "Arthropod pest management in organic crops," Annual Review of Entomology, vol. 52, pp. 57-80, 2007.

[18] R. M. Anderson and R. M. May, "Regulation and stability of host-parasite interactions: I. regulatory processes," Journal of Animal Ecology, vol. 47, no. 1, pp. 219-247, 1978.

[19] P. D. N. Srinivasu and B. S. R. V. Prasad, "Role of quantity of additional food to predators as a control in predator-prey systems with relevance to pest management and biological conservation," Bulletin of Mathematical Biology, vol. 73, no. 10, pp. 2249-2276, 2011. 
[20] H. Zhang, L. Chen, and J. J. Nieto, "A delayed epidemic model with stage-structure and pulses for pest management strategy," Nonlinear Analysis, vol. 9, no. 4, pp. 1714-1726, 2008.

[21] S. Bhattacharyya and D. K. Bhattacharya, "Pest control through viral disease: mathematical modeling and analysis," Journal of Theoretical Biology, vol. 238, no. 1, pp. 177-197, 2006.

[22] B. J. Cardinale, C. T. Harvey, K. Gross, and A. R. Ives, "Biodiversity and biocontrol: emergent impacts of a multi-enemy assemblage on pest suppression and crop yield in an agroecosystem," Ecology Letters, vol. 6, no. 9, pp. 857-865, 2003.

[23] J. M. Tylianakis and C. M. Romo, "Natural enemy diversity and biological control: making sense of the context-dependency," Basic and Applied Ecology, vol. 11, no. 8, pp. 657-668, 2010.

[24] W. W. Murdoch, J. Chesson, and P. L. Chesson, "Biological control in theory and practice," The American Naturalist, vol. 125, no. 3, pp. 344-366, 1985.

[25] S. M. Moore, E. T. Borer, and P. R. Hosseini, "Predators indirectly control vector-borne disease: linking predator-prey and host-pathogen models," Journal of the Royal Society Interface, vol. 7, no. 42, pp. 161-176, 2009.

[26] M. J. Jeger, J. Holt, F. Van Den Bosch, and L. V. Madden, "Epidemiology of insect-transmitted plant viruses: modelling disease dynamics and control interventions," Physiological Entomology, vol. 29, no. 3, pp. 291-304, 2004.

[27] P. W. Ewald and G. De Leo, "Alternative transmission modes and the evolution of virulence," in Adaptive Dynamics of Infectious Diseases, U. Dieckmann, J. A. J. Metz, M. W. Sabelis, and K. Sigmund, Eds., pp. 10-26, Cambridge University Press, New York, NY, USA, 2005.

[28] W. E. Fitzgibbon, M. E. Parrott, and G. F. Webb, "Diffusion epidemic models with incubation and crisscross dynamics," Mathematical Biosciences, vol. 128, no. 1-2, pp. 131-155, 1995.

[29] J. Holt, M. J. Jeger, J. M. Thresh, and G. W. Otim-Nape, "An epidemiological model incorporating vector population dynamics applied to African cassava mosaic virus disease," Journal of Applied Ecology, vol. 34, no. 3, pp. 793-806, 1997.

[30] S. A. Gourley, R. Liu, and J. Wu, "Eradicating vector-borne diseases via age-structured culling," Journal of Mathematical Biology, vol. 54, no. 3, pp. 309-335, 2007.

[31] H.-M. Wei, X.-Z. Li, and M. Martcheva, "An epidemic model of a vector-borne disease with direct transmission and time delay," Journal of Mathematical Analysis and Applications, vol. 342, no. 2, pp. 895-908, 2008.

[32] J. Tumwiine, J. Y. T. Mugisha, and L. S. Luboobi, "A hostvector model for malaria with infective immigrants," Journal of Mathematical Analysis and Applications, vol. 361, no. 1, pp. 139149, 2010.

[33] Z. P. Qiu, Q. K. Kong, X. Z. Li, and M. Martcheva, "The vectorhost epidemic model with multiple strains in a patchy environment," Journal of Mathematical Analysis and Applications, vol. 405, no. 1, pp. 12-36, 2013.

[34] A. A. Lashari and G. Zaman, "Global dynamics of vector-borne diseases with horizontal transmission in host population," Computers \& Mathematics with Applications, vol. 61, no. 4, pp. 745-754, 2011.

[35] M. J. Jeger, F. Van den Bosch, and L. V. Madden, "Modelling virus-and host-limitation in vectored plant disease epidemics," Virus Research, vol. 159, no. 2, pp. 215-222, 2011.

[36] J. G. Schraiber, A. N. Kaczmarczyk, R. Kwok et al., "Constraints on the use of lifespan-shortening Wolbachia to control dengue fever," Journal of Theoretical Biology, vol. 297, pp. 26-32, 2012.
[37] H. Hughes and N. F. Britton, "Modelling the use of Wolbachia to control dengue fever transmission," Bulletin of Mathematical Biology, vol. 75, no. 5, pp. 796-818, 2013.

[38] K. W. Okamoto and P. Amarasekare, "The biological control of disease vectors," Journal of Theoretical Biology, vol. 309, pp. 4757, 2012.

[39] C. S. Holling, "The functional response of predators to prey density and its role in mimicry and population regulations," Memoirs of the Entomological Society of Canada, vol. 45, pp. 360, 1965.

[40] O. Diekmann, J. A. P. Heesterbeek, and J. A. J. Metz, "On the definition and the computation of the basic reproduction ratio $R_{0}$ in models for infectious diseases in heterogeneous populations," Journal of Mathematical Biology, vol. 28, no. 4, pp. 365382, 1990.

[41] P. Van den Driessche and J. Watmough, "Reproduction numbers and sub-threshold endemic equilibria for compartmental models of disease transmission," Mathematical Biosciences, vol. 180, no. 1-2, pp. 29-48, 2002.

[42] J. K. Hale, Ordinary Differential Equations, John Wiley \& Sons, New York, NY, USA, 1969.

[43] H. L. Smith, Monotone Dynamical Systems: An Introduction to the Theory of Competitive and Cooperative Systems, vol. 41 of Mathematical Surveys and Monographs, American Mathematical Society, Providence, RI, USA, 1995.

[44] H. L. Smith, "Cooperative systems of differential equations with concave nonlinearities," Nonlinear Analysis: Theory, Methods \& Applications, vol. 10, no. 10, pp. 1037-1052, 1986.

[45] C. Castillo-Chavez and H. R. Thieme, "Asymptotically autonomous epidemic models," in Mathematical Population Dynamics: Analysis of Heterogeneity, O. Arino and M. Kimmel, Eds., vol. 1 of Theory of Epidemics, pp. 33-50, Wuerz, Winnipeg, Canada, 1995.

[46] J. J. Tewa, V. Y. Djeumen, and S. Bowong, "Predator-prey model with Holling response function of type II and SIS infectious disease," Applied Mathematical Modelling, vol. 37, no. 7, pp. 4825-4841, 2013. 


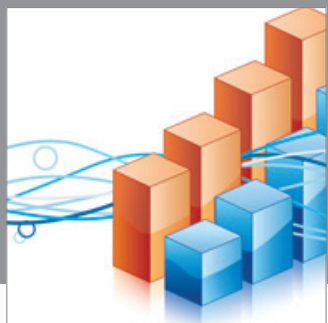

Advances in

Operations Research

mansans

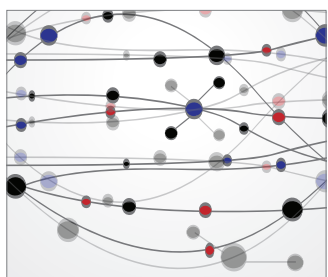

The Scientific World Journal
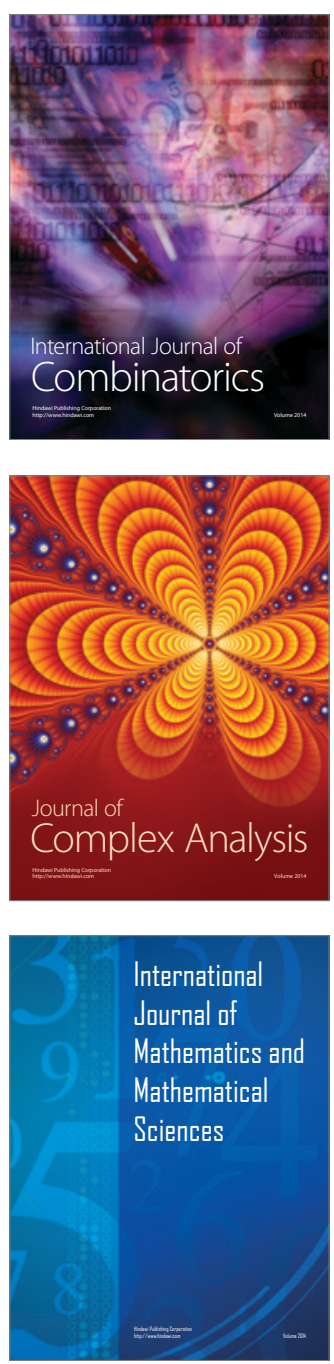
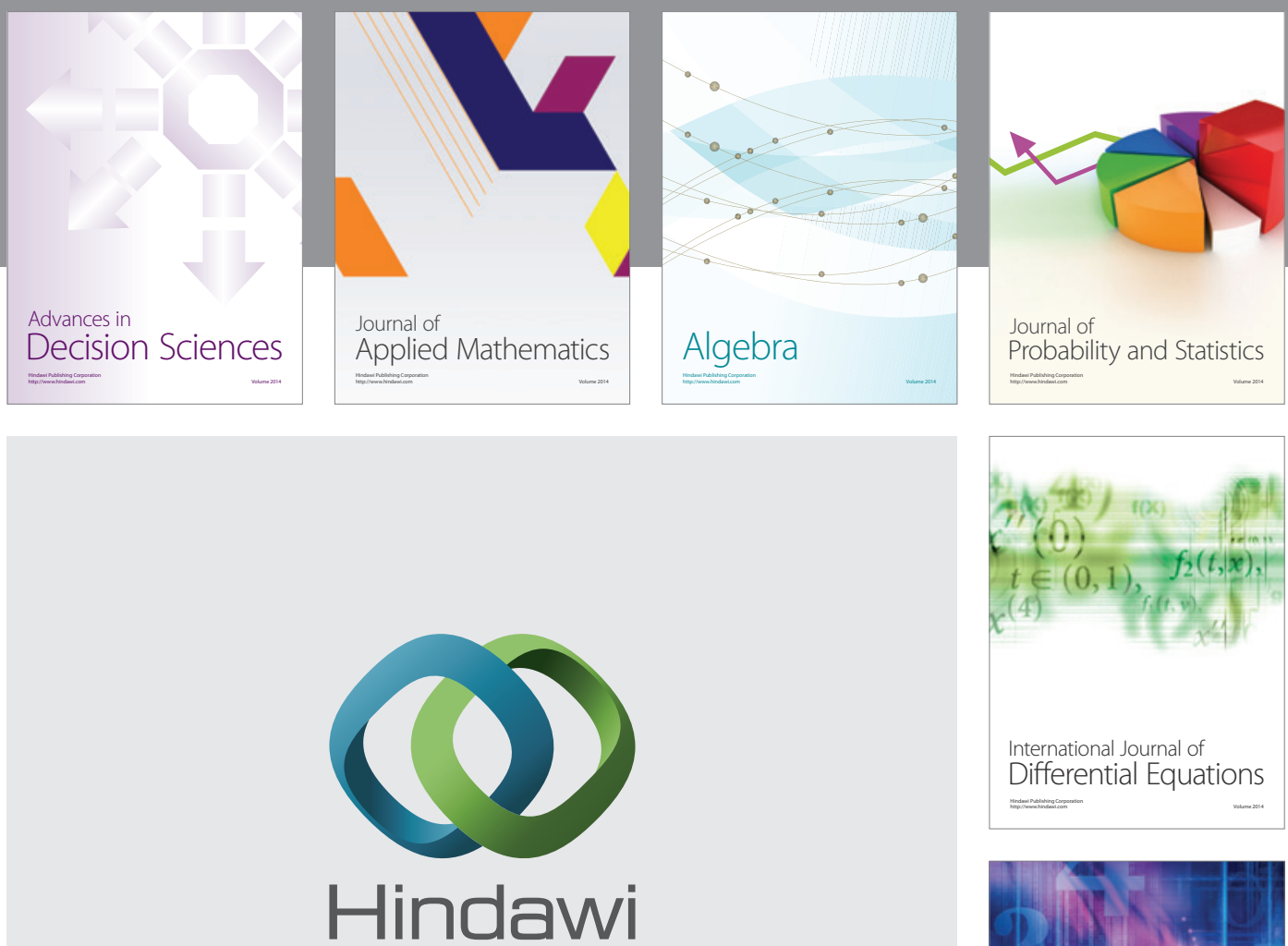

Submit your manuscripts at http://www.hindawi.com
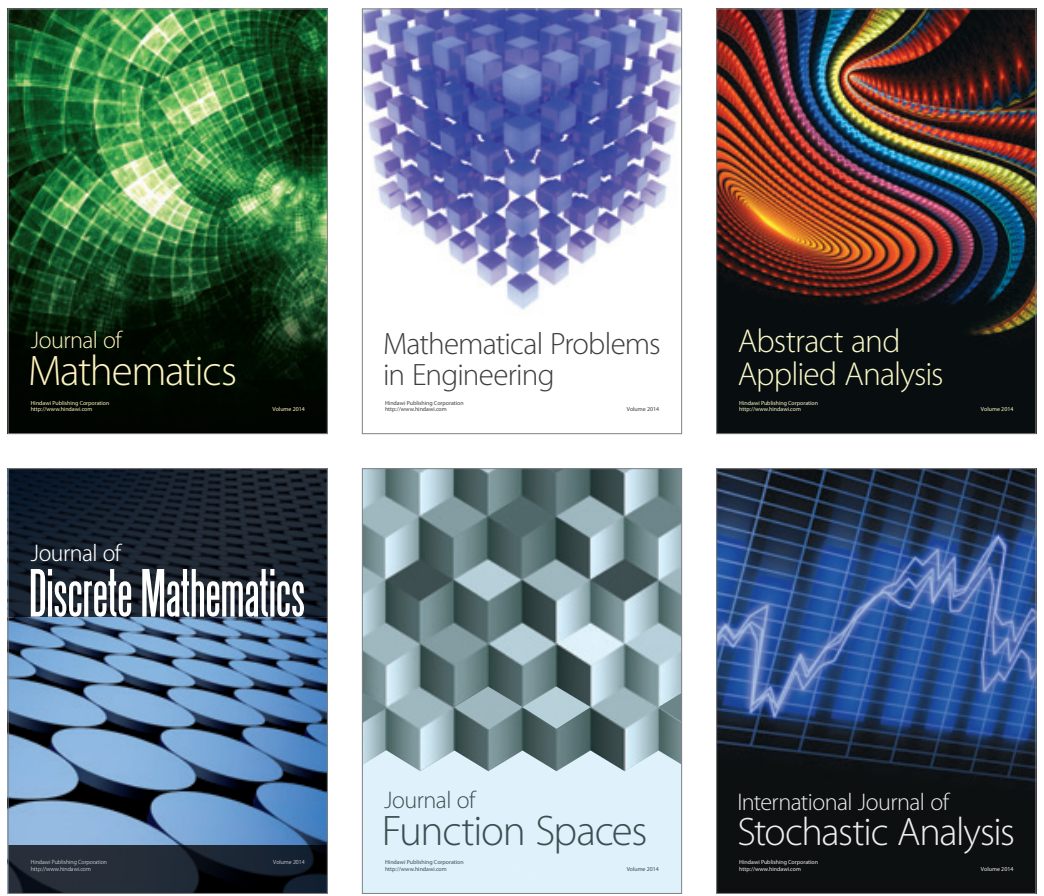

Journal of

Function Spaces

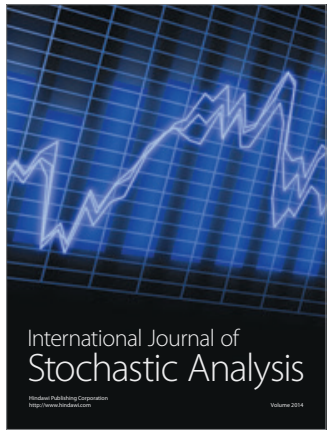

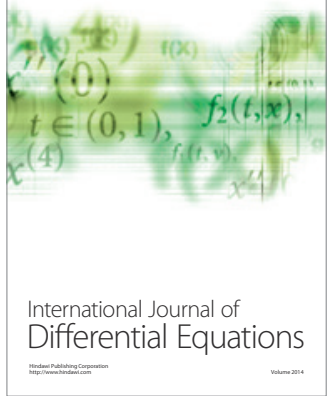
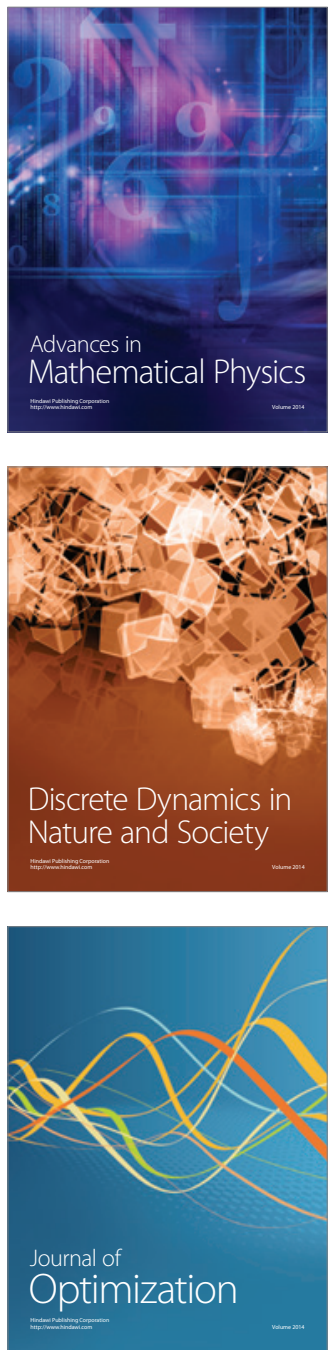